\title{
Feasibility of direct solid sampling for arsenic determination in sulfur-containing active pharmaceutical ingredients by GF AAS
}

\author{
Erico M. M. Flores ${ }^{\mathrm{a}}$, Fabio A. Duarte ${ }^{\mathrm{a}}$, Gabriel T. Druzian ${ }^{\mathrm{a}}$, Mariele S. Nascimento ${ }^{\mathrm{a}}$, Juliano S. Barin ${ }^{\mathrm{b}}$ and Rodrigo C. Bolzan $^{\mathrm{a}, *}$ \\ ${ }^{a}$ Departamento de Química, Universidade Federal de Santa Maria, Santa Maria, RS, Brazil \\ ${ }^{b}$ Departamento de Tecnologia e Ciência dos Alimentos, Universidade Federal de Santa Maria, Santa Maria, RS, Brazil \\ *Corresponding author: rodrigo.bolzan@ufsm.br
}

\begin{abstract}
A method for As determination in sulfur-containing active pharmaceutical ingredients (SC-APIs) by direct solid sampling graphite furnace atomic absorption spectrometry (DSS-GF AAS) was developed. The proposed method was successfully applied to three SC-APIs (hydrochlorothiazide, furosemide and sulfadiazine). Palladium was used as chemical modifier as well as hydrogen during the pyrolysis allowing the direct determination of As in the SC-APIs without interferences caused by gaseous sulfur species. Sample masses (hydrochlorothiazide) from 0.4 to $3 \mathrm{mg}$ were used and calibration with aqueous standard solutions was feasible. The limit of quantification was $0.033 \mu \mathrm{g} \mathrm{g}^{-1}$ and the calibration ranged from 0.1 to $1.6 \mathrm{ng}$ As. Recoveries for As solutions added directly to the solid samples were between 95 and 103\%, showing a good accuracy. The method validation highlighted its robustness, since variation in pyrolysis and atomization temperatures, as well as in $\mathrm{Pd}$ and sample masses, did not change significantly the results. Additional experiments showed that this method can be applied to other SC-APIs (as e.g., furosemide and sulfadiazine). Arsenic concentration in hydrochlorothiazide samples ranged from 0.13 to $0.48 \mu \mathrm{g} \mathrm{g}^{-1}$, while in furosemide and sulfadiazine samples it was from 0.49 and $0.54 \mu \mathrm{g} \mathrm{g}^{-1}$, respectively. The use of DSS-GF AAS does not require previous sample digestion and As could be directly determined in the solid samples providing some advantages, as lower risks of contamination and analyte losses, good accuracy and limits of quantification.
\end{abstract}

Keywords: Arsenic determination; Solid sampling; Graphite furnace atomic absorption spectrometry; Elemental impurities determination; Toxic elements.

\section{Introduction}

The determination of elemental impurities is a critical aspect for the quality control of pharmaceutical products mainly due to their toxicity [1]. In view of the presence of some toxic impurities, the routine quality control of active pharmaceutical ingredients (APIs) by pharmaceutical industry is mandatory [2-4]. Moreover, some contaminants could decrease the stability of APIs even at ultra-trace levels [5]. Among these impurities, arsenic is one of the most relevant due to its toxicity, in special the carcinogenic effect [6]. Arsenic is classified as "class 1" elemental impurity (ICH Q3D) [7], due to the toxicological aspects and the likelihood of occurrence of this element in pharmaceutical products [7]. Therefore, arsenic should be monitored in APIs in order to access its contamination during the treatment. Hydrochlorothiazide and furosemide (Figure 1, A and B) are raw materials widely used in pharmaceutical industry for the production of diuretic and antihypertensive medicines. In the same way, sulfadiazine (Figure 1C) is a raw material used for the production of antibiotics [8]. The common feature among them is that all are sulfur-containing active pharmaceutical ingredients (SC-APIs).

Arsenic determination has been described in many pharmacopoeias as United States Pharmacopoeia (USP) [2], European Pharmacopoeia (EP) [3] and Brazilian Pharmacopoeia (BP) [4]. USP and EP set the maximum arsenic concentration of $1.5 \mu \mathrm{g} \mathrm{g} \mathrm{g}^{-1}$ (for parenteral and oral exposure) in agreement with ICH Q3D [7]. On the other hand, for hydrochlorothiazide and furosemide, BP only establishes the limit to the maximum heavy metal group content (the sum of heavy metals content, in which As is included) as being 10 and $20 \mu \mathrm{g} \mathrm{g}^{-1}$ for hydrochlorothiazide and furosemide, respectively. For sulfadiazine, BP establishes a maximum content of heavy metals of $20 \mu \mathrm{g} \mathrm{g-1}$ and $2 \mu \mathrm{g} \mathrm{g}{ }^{-1}$ of arsenic.

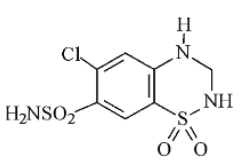

A

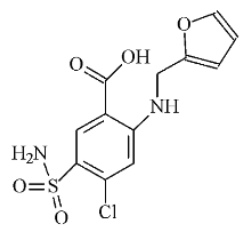

B

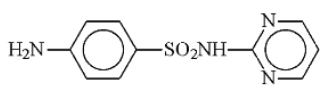

C
Figure 1. Structure of SC-APIs: hydrochlorothiazide (A), furosemide (B), sulfadiazine (C).

Inductively coupled plasma optical emission spectrometry (ICP-OES) and inductively coupled plasma mass spectrometry (ICP-MS) have been recommended for arsenic determination in pharmaceutical products and APIs [4,9-10]. Both techniques allow a rapid and accurate determination of As with low limits of detection (LODs, $\mathrm{ng} \mathrm{g}^{-1}$ range) [11]. However, before the determination by ICP-OES or ICP-MS, the solubilization or digestion of sample matrix is commonly performed by using concentrated acids and under heating $[4,10]$. Unfortunately, this procedure generally is time consuming and prone to contamination and analyte losses [12-14].

On the other hand, As can also be determined by graphite furnace atomic absorption spectrometry (GF AAS), a wellestablished technique for As determination in several types of samples [15-16]. However, the conventional application of GF AAS also involves a previous sample digestion. An alternative to this conventional procedure is by using direct 
solid sampling graphite furnace atomic absorption spectrometry (DSS-GF AAS), that enables the direct determination of As in solid samples using a special device for sample introduction. Using DSS-GF AAS, problems related to unsuitable LODs or difficulties on sample digestion can be solved [12]. This technique has been applied for trace elements determination at low levels in a wide range of samples [17-25] taking into account the following features as $i$ ) it practically avoids sample pretreatment step, ii) low contamination risk, iii) low risk of analyte loss, and $i v$ ) the use of corrosive and hazardous chemicals is avoided. Thus, the use of DSS-GF AAS for As determination at low levels in pharmaceuticals could present advantages over the techniques based on wet digestion for further As determination.

A drawback during arsenic determination when using DSSGF AAS for sulfur-containing matrices is the occurrence of spectral and chemical interferences. Thermal decomposition of sulfur-containing compounds often results in chemical species that show structured or broad-band unspecific background (BG) signals over a wide spectral range [26] bringing difficulties to the acquisition of analytical signals. However, the application of chemical modifiers, careful adjustment of heating program and the use of an efficient BG correction system (e.g., Zeeman effect), generally are able to provide suitable GF AAS measurements [18,27]. Hydrogen has also been proved to be effective to reduce the BG [20,23,28] and/or to improve the effectiveness of Pd as chemical modifier [29], making feasible the analysis by DSS-GF AAS. However, this approach was still not applied to the determination of As in organic SC-APIs.

Thus, a method for direct As determination in SC-APIs by DSS-GF AAS is proposed in this work. Operational parameters were evaluated using hydrochlorothiazide sample and the developed method was applied to other SCAPIs as furosemide and sulfadiazine. The influence of chemical modifiers (palladium and/or hydrogen) was also investigated as well as the possibility of instrument calibration using aqueous standards. Results were compared with those obtained by ICP-OES, ICP-MS and GF AAS, after digestion in closed vessels.

\section{Experimental}

\section{Instrumentation and operational conditions}

Arsenic determination was carried out using a AAS ZEEnit 60 atomic absorption spectrometer (Analytik Jena, Germany) equipped with a Zeeman-effect BG correction system (field strength $0.8 \mathrm{~T}$ ). Transversely heated graphite tubes, a hollow cathode lamp for As (8 mA, $193.7 \mathrm{~nm})$, a device for direct solid sampling (Model SSA-6Z, Analytik Jena) and pyrolytic coated graphite tubes (Analytik Jena, Part Nr. 407-152.023) were used. A spectral bandpass of 0.8 $\mathrm{nm}$ was used and the analytical signals were integrated in 10 s. Background was monitored in peak height. A microbalance model M2P (Sartorius Germany) with resolution of $1 \mu \mathrm{g}$ was used for sample weighing.

Argon $99.996 \%$ of purity (White Martins - Praxair, Brazil) was used for the instruments of ICP-OES, ICP-MS and GF AAS. Hydrogen (99.999\%, White Martins) was used for DSS-GF AAS measurements.
For comparison of the results obtained by the proposed method, the SC-API samples were also digested in quartz closed vessels under microwave radiation (Multiwave 3000, Anton Paar, Austria) and the digests were analyzed by ICPOES, ICP-MS and GF AAS.

Arsenic determination by ICP-OES was performed using a Optima 4300 DV (Perkin Elmer, USA), equipped with a cyclonic spray chamber, a GemCone ${ }^{\circledR}$ nebulizer, and quartz torch with alumina injector tube (2 $\mathrm{mm}$ i.d.). Radiofrequency power was set at $1400 \mathrm{~W}$ and the principal, nebulizer and auxiliary gas flow rates were 15, 0.6 and $0.2 \mathrm{~L}$ $\mathrm{min}^{-1}$, respectively. The wavelength $188.979 \mathrm{~nm}$ was used for data acquisition (three points per peak and two points for BG correction). The instrument was operated using axial view configuration.

The determinations by ICP-MS was performed by using an Elan DRC II (PerkinElmer Sciex, Canada), equipped with a concentric nebulizer (Meinhard Associates, USA), a cyclonic spray chamber and a quartz torch with a quartz injector tube ( $2 \mathrm{~mm}$ i.d.). Radiofrequency power was set at $1300 \mathrm{~W}$ and principal, auxiliary gas and nebulizer gas flow rates were $15,1.2$ and $1.12 \mathrm{~L} \mathrm{~min}^{-1}$, respectively. The $\mathrm{m} / z 75$ was monitored for As. For the analysis by conventional GF AAS the conditions recommended by the manufacturer were used throughout (Analytik Jena AG, Win AAS V 3.13.0 eng, 1998-2004, Jena, Germany).

\section{Samples and Reagents}

Powdered pharmaceutical grade hydrochlorothiazide (samples A, B, C and D) and also furosemide and sulfadiazine (one sample each) were purchased from pharmaceutical industries. Hydrochlorothiazide sample A was used for method development, optimization of heating program and the evaluation of chemical modifiers by DSSGF AAS. These samples were previously dried at $105^{\circ} \mathrm{C}$ for 2 h. Purified water (Milli-Q system - Millipore Corp., USA) was used for preparation of solutions and dilutions. The As reference solutions were prepared by dilution of a stock solution (1000 mg L-1 As in $2 \% \mathrm{HNO}_{3}$, Merck). Concentrated $\mathrm{HNO}_{3}$ was distilled in a sub-boiling system (Milestone, Model duoPUR 2.01 E, Italy). All other reagents were of analytical grade or better (Merck, Germany).

\section{Procedures}

For As determination by DSS-GF AAS, solid samples were weighted directly on the graphite platform, followed by the addition of Pd solution as chemical modifier. Thus, the solid sample plus the chemical modifier were transferred to the graphite tube. Blanks were evaluated by simulating the same steps. Pyrolysis and atomization curves were established with temperatures ranging from 1000 to $1800{ }^{\circ} \mathrm{C}$ and from 2000 to $2600{ }^{\circ} \mathrm{C}$, respectively. The effectiveness of hydrogen as chemical modifier $\left(0.5 \mathrm{~L} \mathrm{~min}^{-1}\right.$ added as additional gas) was evaluated by adding during the pyrolysis step combined with the use of $6 \mu \mathrm{g} \mathrm{Pd}$. The amount of chemical modifier was based on the conditions described in the literature [20]. Additionally, the suitable sample mass range (from 0.1 to $3 \mathrm{mg}$ ) that can be used for As determination was also investigated [30]. 
For As determination by ICP-OES, ICP-MS and GF AAS, hydrochlorothiazide samples were previously digested under microwave irradiation in quartz closed vessels. About $0.3 \mathrm{~g}$ of sample were transferred to the vessels and $6 \mathrm{~mL}$ of concentrated $\mathrm{HNO}_{3}$ were added. Vessels were closed, and the following irradiation program was applied: $10 \mathrm{~min}$ of ramp up to $1400 \mathrm{~W}, 1400 \mathrm{~W}$ for $20 \mathrm{~min}$ and $20 \mathrm{~min}$ at $0 \mathrm{~W}$ for cooling [31]. Afterwards, digests were diluted with water up to $50 \mathrm{~mL}$.

The optimized conditions for As determination in hydrochlorothiazide by DSS-GF AAS were also applied for the analysis of furosemide and sulfadiazine samples.

\section{Method validation and statistical analysis}

Considering that there is no available certified reference material for As with similar composition to hydrochlorothiazide, the accuracy of the proposed method was performed by two approaches: i) recovery tests performed by adding arsenic (from reference solution) to the solid hydrochlorothiazide sample after weighing; ii) comparison with other detection techniques (ICP-OES, ICP$\mathrm{MS}$ and GF AAS) after microwave-assisted digestion. The limit of quantification (LOQ) and the linearity $\left(\mathrm{R}^{2}\right)$ of analytical curve were also evaluated. Robustness was evaluated based on the results obtained after variations in the pyrolysis and atomization temperatures and for the mass of sample and Pd. Repeatability was studied with intra-day and inter-day (intermediate precision) through the relative standard deviation (RSD) after analysis. These parameters were evaluated according to ICH Q2(R1) [32].

Data were analyzed by ANOVA followed by Tukey's test when suitable. Data was considered statistical different when $\mathrm{p}<0.05$.

\section{Results and discussion}

\section{Pyrolysis and atomization curves by DSS-GF AAS for As determination in hydrochlorothiazide}

In all experiments the use of $\mathrm{Pd}(6 \mu \mathrm{g})$ as chemical modifier was performed taking into account its effectiveness in the As determination in a wide variety of samples [20,33]. Preliminary studies showed the need of a cool down step $\left(100{ }^{\circ} \mathrm{C}, 15 \mathrm{~s}\right)$ before atomization to impair further interference caused by matrix vapors formed during the pyrolysis. It is important to point out that since using pyrolysis temperatures of $1600{ }^{\circ} \mathrm{C}(50 \mathrm{~s})$ the spectrometer was not able to set the baseline prior to the atomization, being mandatory the use of the cool down step.

In initial experiments it was attempted to perform the pyrolysis and atomization curves for arsenic in hydrochlorothiazide by DSS-GF AAS. The analytical signals presented high baseline drifting caused by the high $\mathrm{BG}$ signal. As an example, in Figure $2 \mathrm{~A}$ is shown that in the recommended pyrolysis temperature $\left(1300{ }^{\circ} \mathrm{C}\right)$ for arsenic using Pd [34-36] the BG signal was about 1.4 (peak height) and an insufficiently corrected/high noise analytical signal was observed, probably due to the overcorrection effect. The application of higher pyrolysis temperatures resulted in lower BG signals, but the analytical signal also decreased, even using $\mathrm{Pd}$, impairing the As determination (data not shown).

Thus, due to the impossibility to obtain analytical signals without any overcorrection problems, tests using gaseous hydrogen introduced together with argon into the graphite tube during the pyrolysis were performed. The use of gaseous hydrogen was already proposed for pre-reduction of $\mathrm{Pd}$ into the graphite tube forming metallic $\mathrm{Pd}$ in order to increase its efficiency [37] and/or decrease BG signals $[38,39]$. As can be seen in Figure $2 \mathrm{~B}$ the use of hydrogen decreased BG values. The background signal for As determination in solid hydrochlorothiazide by DSS-GF AAS was relatively small $(<0.1$, peak height $)$ and the analytical signal did not present baseline drifting and other overcorrection problems. Therefore, pyrolysis and atomization curves were established with hydrogen addition during the pyrolysis step.
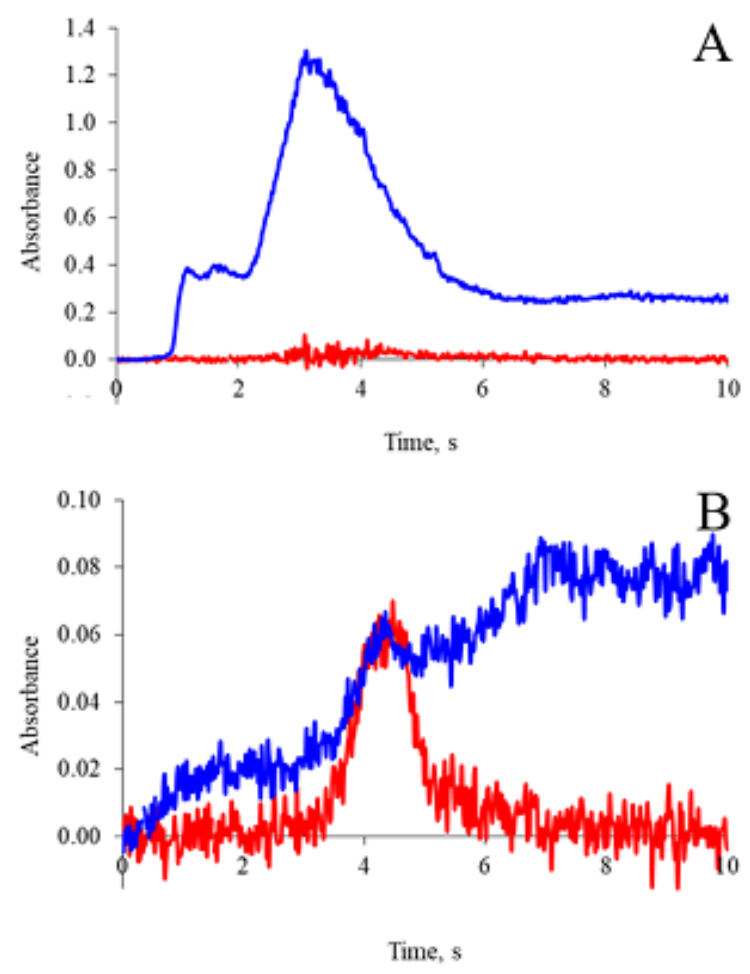

Figure 2: Analytical (red) and BG (blue) signals for arsenic in hydrochlorothiazide by DSS-GF AAS. Pyrolysis at $1300{ }^{\circ} \mathrm{C}$ and atomization at $2400{ }^{\circ} \mathrm{C}$, using $6 \mu \mathrm{g} \mathrm{Pd}$. A: $0.949 \mathrm{mg}$ (no $\mathrm{H}_{2}$ during the pyrolysis step); B: $0.988 \mathrm{mg}\left(0.5 \mathrm{~L} \min ^{-1} \mathrm{H}_{2}\right.$ during the pyrolysis step).

The pyrolysis curve for As determination in solid hydrochlorothiazide by DSS-GF AAS (Figure 3) presented very low $\mathrm{BG}$ signal at temperatures higher than $1100{ }^{\circ} \mathrm{C}$. The best analytical signal was obtained at $1300{ }^{\circ} \mathrm{C}$, once that at higher temperatures, the analytical signals decreased and higher RSDs were observed. For the atomization curve (Figure 3) the BG signals were lower than 0.4 (peak height), being well corrected by the Zeeman-effect correction system. The analytical signals increased up to $2400{ }^{\circ} \mathrm{C}$, presenting a peak shape with no overcorrection and total integration in $10 \mathrm{~s}$ (Figure 2B). For atomization 
temperatures of 2500 and $2600{ }^{\circ} \mathrm{C}$, the analytical signals presented lower precision.

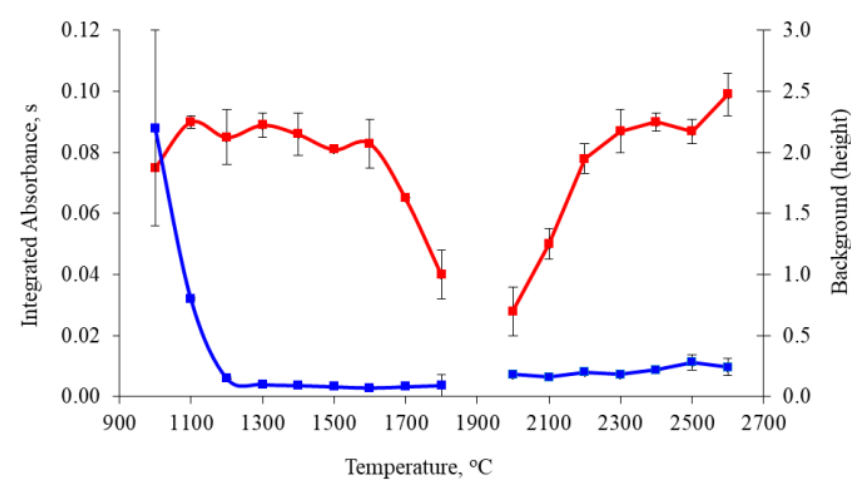

Figure 3: Pyrolysis and atomization curves for $A s$ in hydrochlorothiazide using DSS-GF AAS: analytical (red) and BG (blue, peak height) signals. Pyrolysis at $1300{ }^{\circ} \mathrm{C}$ and atomization at $2400{ }^{\circ} \mathrm{C}$. Vertical bars are the standard deviations $(\mathrm{n}=3)$. Hydrochlorothiazide mass was $1.018 \pm 0.04 \mathrm{mg}, 6 \mu \mathrm{g}$ Pd was used as chemical modifier and hydrogen $0.5 \mathrm{~L} \mathrm{~min}^{-1}$ were used during the pyrolysis step.

Thus, 1300 and $2400{ }^{\circ} \mathrm{C}$ were selected as pyrolysis and atomization temperatures, respectively, for As determination in hydrochlorothiazide by DSS-GF AAS. These temperature parameters were partially in agreement with those reported in previous works suggesting 1300 and $2200{ }^{\circ} \mathrm{C}$ for pyrolysis and atomization temperatures, respectively, for As determination using $\mathrm{Pd}$ as chemical modifier [20,34-36]. Although no memory effects have been observed, a clean-up step $\left(2600{ }^{\circ} \mathrm{C}\right)$ was always applied. The optimized conditions for As determination in hydrochlorothiazide by DSS-GF AAS are shown in Table 1.

\section{Sample mass range}

The sample mass range that could be introduced into the graphite tube under optimized conditions (Table 1) was investigated according to a work previously described in the literature [30].

The effect of sample mass (hydrochlorothiazide) on the analytical signal was assessed by DSS-GF AAS (Figure 4), and sample masses from 0.05 to $3 \mathrm{mg}$ were used. Results show that low sample masses (from 0.05 to $0.4 \mathrm{mg}$ ) produced overestimated results, although masses higher than $0.4 \mathrm{mg}$ showed no influence on the analytical signal. The occurrence of underestimated signals for high samples masses (up to $3 \mathrm{mg}$ ) were not observed [30]. Moreover, further experiments showed that hydrochlorothiazide masses ranging from $0.4 \mathrm{mg}$ up to $3 \mathrm{mg}$ could be used without any interference in the analytical performance.

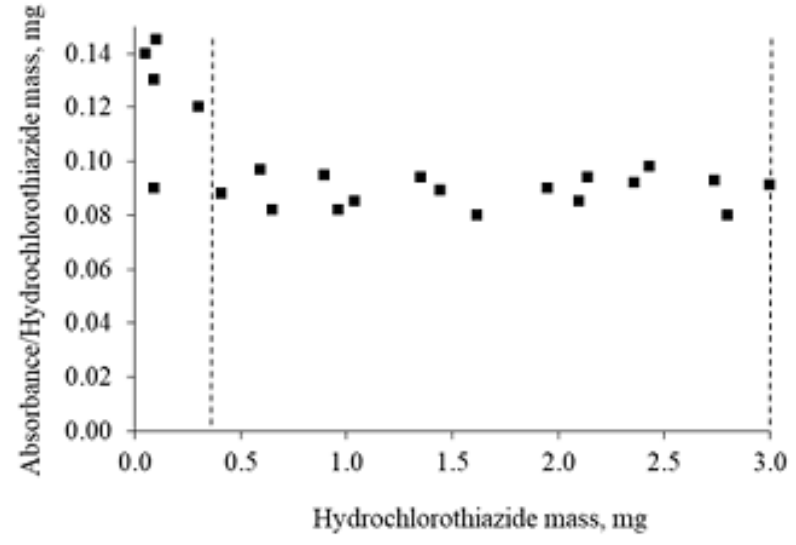

Figure 4: Influence of sample mass on analytical results for As determination by DSS-GF AAS. Dotted lines show the usable mass range.

\section{Sample analysis and method validation}

Calibration was feasible using aqueous reference standard solutions. Thus, the characteristic mass for the proposed method by DSS-GF AAS for As was 20.17 pg (aqueous solution) [34].

The As concentrations in hydrochlorothiazide samples ranged from 0.13 to $0.48 \mu \mathrm{g} \mathrm{g}^{-1}$ (Table 2). The results obtained by the proposed method were not statistically different from those by ICP-MS and GF AAS after acid digestion. Arsenic concentrations in hydrochlorothiazide samples by ICP-OES were lower than the LOD $(3 \sigma, \mathrm{n}=10)$, impairing any comparison.

Accuracy was also evaluated by adding As reference solutions to the solid hydrochlorothiazide (sample A), after weighing and modifier addition, corresponding to As concentration of $0.68,0.96$ and $1.24 \mu \mathrm{g} \mathrm{g}$. Recoveries ranged from 95 to $103 \%$ (RSD below 6\%). The LOQ, calculated using the standard deviation of 10 blank measurements $(10 \sigma, \mathrm{n}=10)$, was $0.033 \mu \mathrm{g} \mathrm{g}{ }^{-1}$. Since the maximum As content allowed by ICH Q3D is $1.5 \mu \mathrm{g} \mathrm{g}^{-1}$ [7], this LOQ was considered suitable and fit for purpose. The linear concentration range of the calibration curve was from 0.1 to $1.6 \mathrm{ng}\left(\mathrm{R}^{2}>0.995\right)$. The robustness of the proposed method was evaluated varying the pyrolysis (1200 to 1400 ${ }^{\circ} \mathrm{C}$ ) and atomization (2300 to $2500{ }^{\circ} \mathrm{C}$ ) temperatures, the $\mathrm{Pd}$ amount (5 to $7 \mu \mathrm{g}$ ) and sample mass $(0.4$ to $3 \mathrm{mg}$ ). After varying all these parameters, the variation in the analytical results were lower than $10 \%$. The repeatability and intermediate precision presented RSD below 10\%. All these parameters were considered suitable for arsenic determination.

\section{Feasibility of the proposed method for As determination in other SC-APIs}

Furosemide and sulfadiazine are well known SC-APIs currently used in therapeutics. These molecules present sulfur atoms, that could lead to interferences when the As determination is performed by DSS-GF AAS, as previously discussed for hydrochlorothiazide (Figure 2). These SCAPIs also require quality control related to elemental impurities/arsenic content [2-4]. 
As can be seen in Figures $5 \mathrm{~A}$ and $5 \mathrm{~B}$, relative high $\mathrm{BG}$ signals were observed when furosemide and sulfadiazine

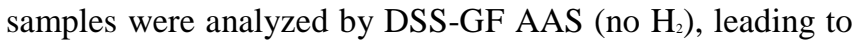

insufficiently corrected/high noise analytical signals, probably due to the overcorrection.

Table 1: Heating program for arsenic determination in hydrochlorothiazide by DSS-GF AAS.

\begin{tabular}{|c|c|c|c|c|c|}
\hline . & $\begin{array}{l}\text { Temperature } \\
\left({ }^{\circ} \mathrm{C}\right)\end{array}$ & $\begin{array}{l}\text { Ramp } \\
\left({ }^{\circ} \mathrm{C} \mathrm{s}^{-1}\right)\end{array}$ & Time (s) & $\begin{array}{l}\text { Inert gas } \\
\left(\mathrm{L} \mathrm{min}^{-1}\right)\end{array}$ & $\begin{array}{c}\text { Hydrogen } \\
\left(\mathrm{L} \mathrm{min}^{-1}\right)\end{array}$ \\
\hline Drying \#1 & 110 & 15 & 30 & 2 & - \\
\hline Drying \#2 & 120 & 15 & 15 & 2 & - \\
\hline Pyrolysis \#1 & 1300 & 400 & 20 & 2 & 0.5 \\
\hline Pyrolysis \#2* & 100 & 0 & 15 & 2 & - \\
\hline Auto zero & 100 & 0 & 6 & - & - \\
\hline Atomization & 2400 & 3000 & 10 & - & - \\
\hline Clean out & 2600 & 3000 & 4 & 2 & - \\
\hline
\end{tabular}

*Cool down step

Table 2: Results for the determination of arsenic in hydrochlorothiazide by the proposed DSS-GF AAS method (values in $\mu \mathrm{g} \mathrm{g}^{-1}$, mean and standard deviation), and by ICP-OES, ICP-MS and GF AAS after digestion ( $\geq 3$ ).

\begin{tabular}{|c|c|c|c|c|}
\hline Samples & DSS-GF AAS & ICP-OES & ICP-MS & GF AAS \\
\hline \multicolumn{5}{|c|}{ Hydrochlorothiazide } \\
\hline A & $0.40 \pm 0.03$ & $<0.9 *$ & $0.40 \pm 0.05$ & $0.36 \pm 0.05$ \\
\hline B & $0.13 \pm 0.01$ & $<0.9 *$ & $0.12 \pm 0.03$ & $<0.16^{* *}$ \\
\hline $\mathrm{C}$ & $0.39 \pm 0.05$ & $<0.9 *$ & $0.39 \pm 0.06$ & $0.35 \pm 0.06$ \\
\hline $\mathrm{D}$ & $0.48 \pm 0.03$ & $<0.9^{*}$ & $0.45 \pm 0.06$ & $0.38 \pm 0.05$ \\
\hline Furosemide & $0.49 \pm 0.04$ & $<0.9 *$ & $0.50 \pm 0.05$ & $0.46 \pm 0.06$ \\
\hline Sulfadiazine & $0.54 \pm 0.04$ & $<0.9 *$ & $0.51 \pm 0.06$ & $0.52 \pm 0.05$ \\
\hline
\end{tabular}

* LOD by using ICP-OES; ** LOD by using GF AAS
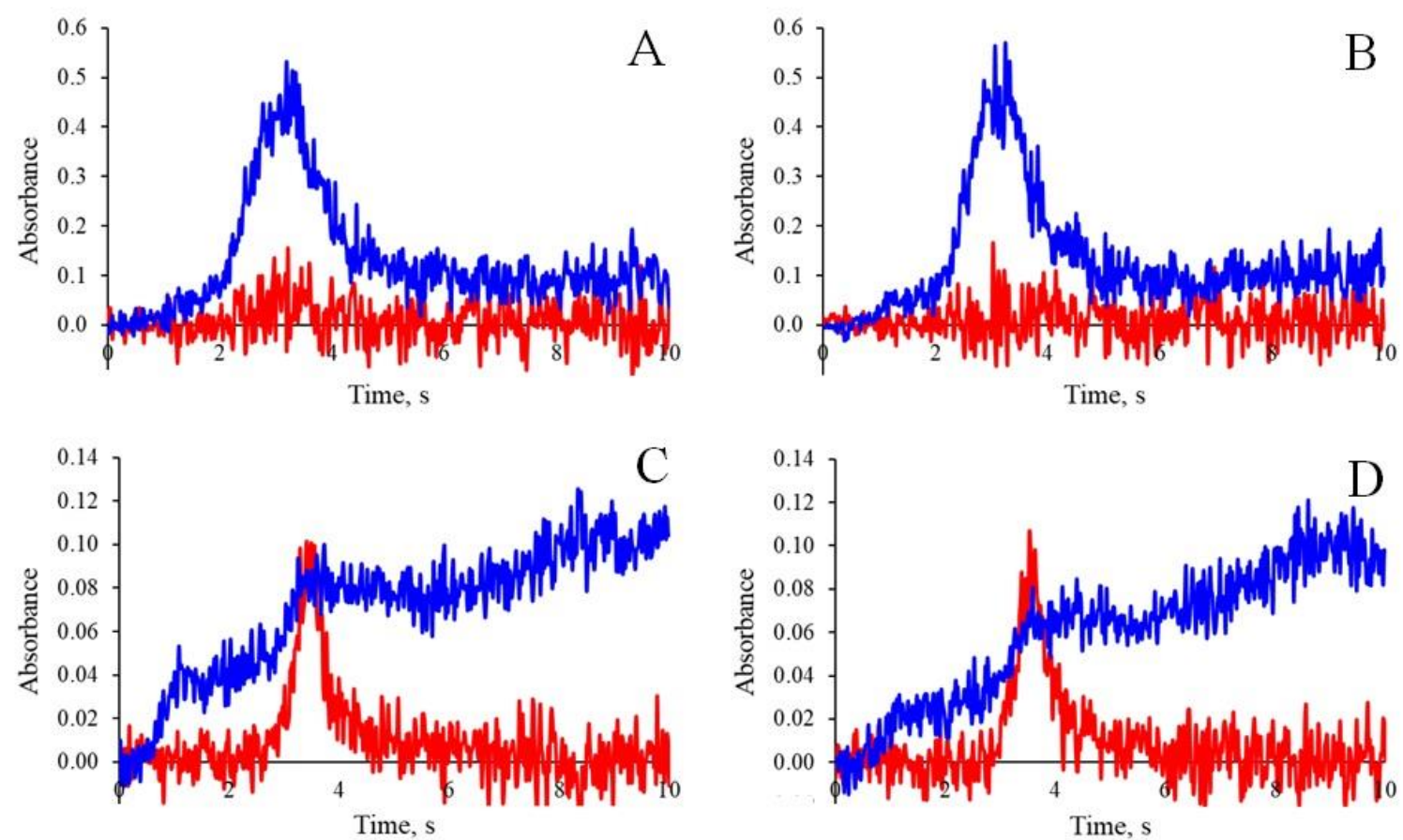

Figure 5: Analytical (red) and BG (blue) signals for arsenic in SC-APIs by DSS-GF AAS. Pyrolysis at $1300{ }^{\circ} \mathrm{C}$ and atomization at $2400{ }^{\circ} \mathrm{C}$, using $6 \mu \mathrm{g}$ Pd. A: $1.012 \mathrm{mg}$ furosemide (no $\mathrm{H}_{2}$ during the pyrolysis step); B: $1.030 \mathrm{mg}$ sulfadiazine (no $\mathrm{H}_{2}$ during the pyrolysis step); $\mathrm{C}$ : 1.042 $\mathrm{mg}$ furosemide $+0.5 \mathrm{~L} \mathrm{~min}^{-1} \mathrm{H}_{2}$ during the pyrolysis step; D: $1.023 \mathrm{mg}$ sulfadiazine $+0.5 \mathrm{~L} \mathrm{~min}^{-1} \mathrm{H}_{2}$ during the pyrolysis step. 
On the other hand, when gaseous $\mathrm{H}_{2}$ was used during the pyrolysis step, the analytical signals for As were acquired without deformations and totally integrated in $10 \mathrm{~s}$. In addition, the background signals were lower than 0.15 (peak height) and were totally correct (Figures 5, C and D). This fact supports that arsenic determination in these sulfur containing substances by DSS-GF AAS is possible if gaseous hydrogen is used during pyrolysis.

In addition, applying the optimized conditions for arsenic determination in hydrochlorothiazide by DSS-GF AAS (Table 1 and discussion in sections 3.2 and 3.3), the arsenic concentration in furosemide and sulfadiazine samples were 0.49 and $0.54 \mu \mathrm{g} \mathrm{g-1}$, respectively (Table 2 ). As also observed for hydrochlorothiazide samples, the arsenic concentrations were not statistically different from those by ICP-MS and GF AAS after acid digestion and the results by ICP-OES were lower than the LOD.

The proposed method by DSS-GF AAS for As determination in SC-APIs presented similar LOQ to those found in literature for other APIs using ICP-MS after sample digestion (MIC/wet digestion) [13,31]. However, DSS-GF AAS allowed the use of lower sample amounts $(<3 \mathrm{mg})$, contrarily to other methods that required previous sample digestion of higher sample amounts (from 80 up to $500 \mathrm{mg}$ ) $[13,31]$. In addition, the time-consuming sample preparation step and the use of concentrated reagents (i.e. $\mathrm{HNO}_{3}, \mathrm{H}_{2} \mathrm{O}_{2}$ ) is avoided. In the proposed method, the heating cycle (determination) was performed in less than 2 minutes (Table 1 ), and if the other analytical procedures (sample weighting, modifier addition, etc.) are included the total time for a determination was only 5 minutes. In this way, sample preparation step is avoided and the results could be obtained faster using DSS-GF AAS.

\section{Conclusion}

The DSS-GF AAS method for As determination in SC-APIs was feasible. The conventional procedure involves, prior to determination by plasma-based techniques (ICP-OES/ICPMS), sample digestion using concentrated acids and at high temperatures, characterized as a time-consuming process. In this work a simple procedure by using solid sample (no digestion was required) by GF AAS using Pd and hydrogen as chemical modifiers proved to be suitable for direct As determination in these kind of sample, avoiding background overcorrection problems.

\section{Conflict of interest statement}

The authors declare no conflicts of interest.

\section{Acknowledgements}

The authors are grateful to Coordenação de Aperfeiçoamento de Pessoal de Nível Superior (CAPES/Brazil) Finance Code 001, Conselho Nacional de Desenvolvimento Científico e Tecnológico (CNPq/Brazil) $\left(\mathrm{N}^{\circ} 311753 / 2017-5\right)$, and Fundação de Amparo à Pesquisa do Estado do Rio Grande do Sul (FAPERGS/Brazil) (No 17/2551-0000971-1) for supporting this study.

\section{References}

1. Balaram, V., Recent advances in the determination of elemental impurities in pharmaceuticals - Status, challenges and moving frontiers. TRAC-Trend Anal Chem 2016; 80:83-95.

2. General chapter <232> Elemental Impurities - limits: 2nd supplement of USP 41-NF 30, 2018.

3. European Pharmacopoeia (EP), 9.3, section 5.20. Elemental Impurities, 2017.

4. Brazilian Pharmacopoeia, 5th Ed., v2, Agência Nacional de Vigilância Sanitária, Brasília, 2010.

5. Fliszar, K.A., Walker, D., Allain, L., Profiling of metal ions leached from pharmaceutical packaging materials, J Pharm Sci Technol, 2006; 60:337-342.

6. Fowler, B.A., Chou, S.C.H.J., Jones, R.L., Sullivan Jr., D.W., Chen, C.J.. Chapter 28 - Arsenic. In: Handbook on the Toxicology of Metals (Fourth Edition), Academic Press; 2015, Volume II, p. 581-624.

7. The international conference on harmonization of technical requirements and registration of pharmaceuticals for human use (ICH), Guideline for elemental impurities Q3D; 2013.

8. Bardal, S.K., Waechter, J.E., Martin, D.S., Applied pharmacology. Saunders (e-book), 2011.

9. Wang, T., Jane, W., Robert, H., Xiujuan, J., Richard, S.E., A multi-element ICP-MS survey method as an alternative to the heavy metals limit test for pharmaceutical materials. J Pharm Biomed Anal, 2000; 23:867-890.

10. General chapter <233> elemental impurities procedures: 2nd supplement of USP 35-NF 30, 2012.

11. Hou, X., Jones, B.T., Meyers, R.A. (Editor). Encyclopedia of Analytical Chemistry, Chichester:John Wiley \& Sons Ltd, 2000; p. 9468-9485.

12. Vale, M.G.R., Oleszczuk, N., dos Santos, W.N.L., Current status of direct solid sampling for electrothermal atomic absorption spectrometry - A critical review of the development between 1995 and 2005. Appl Spectrosc Rev, 2006; 41:377-400.

13. Barin, J.S., Tischer, B., Picoloto, R.S., Antes, F.G., Silva, F.E.B., Paula, F.R., Flores, E.M.M., Determination of toxic elements in tricyclic active pharmaceutical ingredients by ICP-MS: a critical study of digestion methods. J Anal At Spectrom, 2014; 29:352-358. 
14. Barin, J.S., Mello, P.A., Mesko, M.F., Duarte, F.A., Flores, E.M.M., Determination of elemental impurities in pharmaceutical products and related matrices by ICPbased methods: a review. Anal Bioanal Chem Anal Bioanal Chem, 2016; 408:4547-4566.

15. Viitak, A., Volynsky, A.B., Simple procedure for the determination of $\mathrm{Cd}, \mathrm{Pb}, \mathrm{As}$ and $\mathrm{Se}$ in biological samples by electrothermal atomic absorption spectrometry using colloidal Pd modifier. Talanta, 2006; 70:890-895.

16. Volynsky, A.B., Wennrich, R., Efficiency of Pt and Ir modifiers for the simultaneous determination of As, Se and $\mathrm{In}$ in a sodium sulphate matrix by electrothermal atomic absorption spectrometry. Talanta; 2003; 59:277286.

17. Dong, H.M., Krivan, V., Welz, B., Schlemmer, G., Determination of trace impurities in titanium dioxide by slurry sampling electrothermal atomic absorption spectrometry. Spectrochim Acta Part B, 1997; 52:17471762.

18. Bolzan, R.C., Rodrigues, L.F., Mattos, J.C.P., Dressler, V.L., Flores, E.M.M., Chromium determination in pharmaceutical grade barium sulfate by solid sampling electrothermal atomic absorption spectrometry with Zeeman effect background correction. Talanta, 2007; 74:119-124.

19. Resano, M., Briceño, J., Aramendía, M., Belarra, M.A., Solid sampling-graphite furnace atomic absorption spectrometry for the direct determination of boron in plant tissues. Anal Chim Acta, 2007; 582:214-222.

20. Bolzan, R.C., Moraes, D.P., Mattos, J.C.P., Dressler, V.L., Flores, E.M.M., Arsenic determination in pharmaceutical grade barium sulfate using direct solid sampling electrothermal atomic absorption spectrometry. J Braz Chem Soc, 2010; 21:686-693.

21. Mattos, J.C.P., Rodrigues, L.F., Flores, E.M.M., Krivan, V., Determination of trace impurities in aluminum nitride by direct solid sampling graphite furnace atomic absorption spectrometry. Spectrochim Acta Part B, 2011; 66:637-643.

22. Rodrigues, L.F., Mattos, J.C.P., Bolzan, R.C., Flores, E.M.M., Duarte, F.A., Determination of trace elements in raw material for polyurethane production using direct sampling graphite furnace atomic absorption spectrometry. J Anal At Spectrom, 2014; 29:324-331.

23. Soares, B.M., Santos, R.F., Bolzan, R.C., Muller, E.I., Primel, E.G., Duarte, F.A., Simultaneous determination of iron and nickel in fluoropolymers by solid sampling high-resolution continuum source graphite furnace atomic absorption spectrometry. Talanta, 2016; 160:454460 .
24. Santos, R.F., Carvalho, G.S., Duarte, F.A., Bolzan, R.C., Flores, E.M.M., High purity polyimide analysis by solid sampling graphite furnace atomic absorption spectrometry. Spectrochim Acta Part B, 2017; 129:4248.

25. Seeger, T.S., Machado, E.Q., Flores, E.M.M., Mello, P.A., Duarte, F.A., Direct sampling graphite furnace atomic absorption spectrometry - feasibility of $\mathrm{Na}$ and $\mathrm{K}$ determination in desalted crude oil. Spectrochim Acta Part B, 2018; 141:28-33.

26. Lemme, M., Katskov, D.A., Tittarelli, P., Atomic and molecular spectra of vapors evolved in graphite furnace. Part 7: Alkaline metal sulfates and sulfides. Spectrochim Acta Part B, 2004; 59:115-124.

27. Heitmann, U., Schütz, M., Becker-Ro $\beta$, H., Florek, S., Measurements on the Zeeman-splitting of analytical lines by means of a continuum source graphite furnace atomic absorption spectrometer with a linear charge coupled device array. Spectrochim Acta Part B, 1996; 51:10951105 .

28. Hornung, M., Krivan, V., Solid sampling electrothermal atomic absorption spectrometry for analysis of highpurity tungsten trioxide and high-purity tungsten blue oxide. Spectrochim Acta Part B, 1999; 54:1177-1191.

29. L'vov, B.V., Interpretation of atomization mechanisms in electrothermal atomic absorption spectrometry by analysis of the absolute rates of the processes. Spectrochim Acta Part B, 1997; 52:1-23.

30. Belarra, M.A., Crespo, C., Martínez-Garbayo, M.P., Castillo, J.R., Direct determination of metals in solid samples by graphite-furnace atomic absorption spectrometry: Does sample mass influence the analytical results? Spectrochim Acta Part B, 1997; 52:1855-1860.

31. Muller, A.L.H., Oliveira, J.S.S., Mello, P.A., Muller, E.I., Flores, E.M.M., Study and determination of elemental impurities by ICP-MS in active pharmaceutical ingredients using single reaction chamber digestion in compliance with USP requirements. Talanta, 2015; 136:161-169.

32. ICH. Q2(R1). Validation of Analytical Procedures: Text and Methodology (2005).

33. Zhe-Ming, N., Xiao-Quan, S., The reduction and elimination of matrix interferences in graphite furnace atomic absorption spectrometry. Spectrochim Acta Part B, 1987; 42:937-949.

34. Volynsky, A.B., Comparative efficacy of platinum group metal modifiers in electrothermal atomic absorption spectrometry. Spectrochim Acta Part B, 2004; 59:17991821. 
35. Volynsky, A.B., Wennrich, R., Comparative efficiency of $\mathrm{Pd}, \mathrm{Rh}$ and $\mathrm{Ru}$ modifiers in electrothermal atomic absorption spectrometry towards simultaneous determination of As, Se and In in a sodium sulphate matrix. J Anal At Spectrom, 2001; 16:179-187.

36. Volynsky, A.B., Wennrich, R., Mechanisms of the action of platinum metal modifiers in electrothermal atomic absorption spectrometry: aims and existing approaches. Spectrochim Acta Part B, 2002; 57:1301-1316.

37. Rettberg, T.M., Beach, L.M., Peak profile characteristics in the presence of palladium for graphite furnace atomic absorption spectrometry. J Anal At Spectrom, 1989; 4:427-432.

38. Hornung, M., Krivan, V., Determination of trace impurities in high purity tungsten by direct solid sampling electrothermal atomic absorption spectrometry using a transversely heated graphite tube. Anal Chem, $1998 ; 70: 3444-3451$.

39. L'vov, B.V., Interpretation of atomization mechanisms in electrothermal atomic absorption spectrometry by analysis of the absolute rates of the processes. Spectrochim Acta Part B, 1997; 52:1-23. 